\title{
Modeling Medical Ethics through Intelligent Agents
}

\author{
José Machado $^{1}$, Miguel Miranda ${ }^{1}$, António Abelha ${ }^{1}$, José Neves ${ }^{1}$, \\ and João Neves ${ }^{2}$ \\ 1 Universidade do Minho \\ Departamento de Informática Braga, \\ Portugal \\ \{miranda, jmac, abelha, jneves\}@di.uminho.pt \\ 2 Centro Hospitalar de Vila Nova de Gaia e Espinho, Portugal \\ j_neves@hotmail.com
}

\begin{abstract}
The amount of research using health information has increased dramatically over the last past years. Indeed, a significative number of healthcare institutions have extensive Electronic Health Records (EHR), collected over several years for clinical and teaching purposes, but are uncertain as to the proper circumstances in which to use them to improve the delivery of care to the ones in need. Research Ethics Boards in Portugal and elsewhere in the world are grappling with these issues, but lack clear guidance regarding their role in the creation of and access to EHRs. However, we feel we have an effective way to handle Medical Ethics if we look to the problem under a structured and more rational way. Indeed, we felt that physicians were not aware of the relevance of the subject in their pre-clinical years, but their interest increase when they were exposed to patients. On the other hand, once EHRs are stored in machines, we also felt that we had to find a way to ensure that the behavior of machines toward human users, and perhaps other machines as well, is ethically acceptable. Therefore, in this article we discuss the importance of machine ethics and the need for machines that represent ethical principles explicitly. It is also shown how a machine may abstract an ethical principle from a logical representation of ethical judgments and use that principle to guide its own behavior.
\end{abstract}

Keywords: Morality, intelligent agents, medical ethics.

\section{Introduction}

Ethics is focused on moral goods rather than natural goods. However, both moral and natural goods are equally relevant and have to be taken under consideration. Morals are created by and define society, philosophy, religion or individual conscience, usually associated with the fundamental questions concerning the complexities of the human soul [1. Several forms of ethics have been approached, namely the ones:

(C) IFIP International Federation for Information Processing 2009 
- Applied ethics, i.e. ethics seeks to address questions such as how a moral outcome can be achieved in a specific situation;

- Normative ethics, i.e. how moral values should be determined;

- Descriptive ethics, i.e. what morals people actually abide by;

- meta-ethics, i.e. what the fundamental nature of ethics or morality is, including whether it has any objective justification; and

- Moral psychology, i.e. how moral capacity or moral agency develops and what its nature is.

The role of computers is rapidly evolving from that of passive cipher to that of active participants in the trading process, which lead us to an imperious need of analysing the questions of morality. In Philosophy, morality has different meanings, namely [2]

- A code of conduct which is held to be authoritative in matters of right and wrong;

- An ideal code of conduct, one which would be espoused in preference to alternatives by all rational people, under specified conditions; and

- A synonymous of ethics, the systematic philosophical study of the moral domain.

On the other hand, interoperabiliy in healthcare units is defined as the ability to move clinical data from place to place. Bringing interoperabity to these facilities it is possible to reduce costs and give to clinical and medical staff more powerful tools for patient assistance, in particular in the decision support and problem solving procedures. In Medicine, physicians and nurses have daily to deal with incomplete information, which in association with moral judgements and emotivism, turn decisions sometimes wrong, slow, expensive or unacceptable. This leads us to the need of defining formalisms to identify and evaluate morality and ethics in Medicine.

Medical ethics is primarily a field of applied ethics, the study of moral values and judgments as they apply to Medicine, in particular the examination of particular issues that are matters of moral judgments and morally correct behavior in various fields. Medical ethics encompasses its practical application in clinical settings as well as work on the fields of History, Philosophy, Theology, and Sociology. Medical ethics tends to be understood narrowly as an applied professional ethics, whereas bioethics appears to have worked more expansive concerns, touching upon the philosophy of science and the critique of biotechnology. The two fields often overlap and the distinction is more a matter of style than professional consensus. Medical ethics shares many principles with other branches of healthcare ethics, such as nursing ethics.

Some attributes that may apply to Medical Ethics are depicted below [2]:

- Autonomy,i.e. the patient has the right to refuse or choose their treatment;

- Beneficence, i.e. a practitioner should act in the best interest of the patient;

- Non-maleficence, i.e. "first, do no harm"; 
- Justice, i.e. concerns the distribution of scarce health resources, and the decision of who gets what treatment;

- Dignity, i.e. the patient (and the person treating the patient) have the right to dignity; and

- Truthfulness and honesty, i.e. the concept of informed consent has increased in importance in the last few years.

Those parameters must be quantified and its importance can not be subestimated in the decision making process. All the ethical questions around virtual entities or agents, have to be taken under a practical perspective and are related with the embedded environment. This study has been performed before in terms of electronic commerce, considering the case of the legal and ethical context of contract made by means of intelligent agents 3. 4]. Nonetheless, there exists the need to undergo a particular approach when considering morally dubious areas, where every little may have great moral consequences. This is the case of Medicine, where interoperability and decision support are presently in continuous analysis and development. Following this thread of thought and taking in consideration the state of the art of the Agent Oriented Paradigm, it will be analyzed in this study the moral context of agents, discussing the possibility of an agent at a given state of development, have the moral capacity and legal responsibility for actions.

\section{Intelligent Agents and Medical Ethics}

In a healthcare unit, intelligent agents can be used as a mean towards the integration of different services and the software being used. Within this system, different intelligent agents, autonomously and adaptively, defend individually or by means of cooperation their interests and objectives. They concentrate vital functions of the healthcare unit, improving the quality-of-service and the people quality-of-life. As part of this system there exists different agents which, by different forms, support the medical research, having the capacity to interact with its environment and evolve, acquiring new methodologies and information to improve their own qualities and competence, i.e. to solve different problems according to its duties.

For example, a physician, when analyzing an exam received from a computerized tomography, is presented instead of thousand of pictures, a smaller number of pictures selected by intelligent agents. In light of the selected images, it was not possible to detect any anomaly. Meanwhile, in the group of selected images missed a small set of pictures which evidentiated the existence of small metastasis which might have changed the diagnose. This case was misdiagnosed by influence of the agents. The physician taked a decision which ultimately had moral and legal consequences. This decision revealed itself as a bad help to the diagnose, placing at stake a human life.

Another important topic in medical ethics is the concept of futility. What should be done if there is no chance that a patient will survive but the family 
members insist on advanced care? And what should be made if a patient is in a Intensive Care Unit, using a bed that is necessary to save another patient with more chance to survive? Rational decisions can be taken to solve this particular problem, following legal or practical rules, either by physicians or by intelligent agents. But who will be responsible for taking such moral decisions?

Facing such situations, several questions and doubts arise, namely: What is or defines a Moral Agent? Is an intelligent agent a Moral Agent? Will these agents have at any point in time either the capacity and ability to take moral decisions or being capable to handle with decisions which carry a great ethical dilemma? Which are the legal and moral responsibilities in an agent based system?

The present period, or step in a process or development in Artificial Intelligence (AI) is still far from the usual scenarios imagined by science fiction. However, it is becoming an embedded characteristic in aplications of different areas, from Commerce to Medicine. Indeed, AI techniques which imbue software systems with a considerable degree of intelligence, autonomy and proactivity, and the ability to adapt to the environment being populated are growing, being essential to attain a superior level of utility and interactivity. Infact, it urges the necessity to evaluate and regulate the scope of the capacities of this software systems, either when they are called to execute different tasks or to take decision which may have any arguable moral value. The field of ethics associated to non-organic entities, Machine Ethics, thereby lacks of a more practical oriented and cautious reflexion, that will analyse the state of the art of AI in all its vast extension. It will be then possible to defined moral competences and restrictions to its use in any environment, where morality and reputation are to be questionable.

\section{Intelligent Agents in Medicine}

The requirements of software applications for the healthcare arena, although being rather similar to those of other areas, develops in a completely different dimension due to the value inherent to the moral good, i.e. the health condition of a human being. All agents, either human beings or software agents need to be aware of the immeasurable value of an human life and the ethical complexity existing when dealing with this specific good. As information systems continue to disseminate and strengthen in the healthcare sector, the significance of their use increases and so does their moral responsibilities, i.e. a great part of the scope of intervention of agents in this area carries a moral context and ethical responsibility, which it is made aware, even in software artifacts that inevitably will be designed to automatize and manage the larger loading of information generated by medical practices and underlying activities. In fact, this amount of information is so huge that it becomes impracticable to store and extract any sort of knowledge, without the use of computational methods and AI based techniques.

From the different computational paradigms in use in AI, Agent Oriented Programing has pursued a sheer growth considering the level and number of the 
available systems, being capable of integrating other technologies and techniques for problem solving such as Neural Networks or Case Based Reasoning. An individual agent or a network of agents based on different communities of agent possess a class of characteristics that allows them to be independent from the will, desires and objectives of other virtual agents or human beings, granting a certain degree, although limited, of individuality [5]. On the other hand, an agent method cannot be invoked by other than the entity itself, being determined by its will and degree of responsibility. Only the agent is in charge of its own behavior. Regardless, the use of learning techniques from AI, enable the agent to contextualize and evolve dynamically, making the underlying behaviors dependent of the environment, as well as from other circumstances, which may go out of the scope of its initial parameterization. This possibility rises issues concerning the ethical and legal responsibility of the agent owner, in line with the characterization of intelligent agents as autonomous, self-learning and dynamic entities.

The distributed and heterogeneous nature of this environment, makes the best use of this technology [6], which is being applied to different services and situations, going from heterogeneous system integration to decision support systems 7. A great effort of academic and corporate synergies allowed the use of intelligent agents in several medical centers which aggregate several hospitals and health units, which use an Agency for Integration, Archive and Diffusion of Medical Information (AIDA), an agent based software artifact, that intends to integrate and agregate information from different systems and locations [8].

On the other hand, the use of intelligent agents for integration of systems may not seem to hold a great deal of ethical significance. However,although these tools improve the security and functionality of the medical information as a whole, the consequences of the loss or adulteration of clinical information or the permissiveness towards this sort of actions, carries a unmeasurable ethical and moral value. A lot more can be said about the decision support systems whose action, although being in support of a decision, contributes indirectly to the diagnose and the treatment of patients. Taken these situations into consideration, it becomes essential an objective discussion about the capacities and characteristics of these systems, in order to define the moral competences of an intelligent agent. This characterization is vital as weell as the need of practical guidelines and rules or ethical conduct for the development and behavior of this sort of systems, so that the quality of the services provided may improve.

\section{Moral Capacity}

A moral agency is defined by the moral requisites that drive its behavior. In this way, the underlaying concept of a Moral Agent (MA), relies on the existence of moral premisses that rule its behavior, differentiating good from evil. It is important not to misunderstand the concept of MA with Moral Patient (MP). While the first has moral obligations, a MP is an entity in which moral rights speak for themselves. Moral agents are in most of the cases moral patients, 
however this relation is non-reciprocal, as the discussion on delimitating the grounds of MA considers that only a part of MP are in fact capable of being MA. An adult is a MA although a recently born child is solely a MP, being capable, however, of becoming one during his/her life time [9. This statement that an entity may become during its life time a MA, is indeed very important, once it allows, in an analogous way, to state that an agent, at a given moment, acquire such a property. It is necessary to understand what is a intelligent software agent and which are the characteristics that will allow it to become a MA.

According to Wooldridge, an agent embodies a computational system capable of revealing an autonomous and flexible action, developed in a determined universe of discourse. The flexibility of the agent is related with its capacity of reaction, initiative, learning and socialization [10]. Although the definition may not be considered an universal one, for an organic or software based entity, there exits two levels of intrinsic characteristics, which define in a weak or strong form, whether or not that entity is an intelligent agent. On the one hand, the weak notion of agent represents the minima characteristics of an agent, centering in its capacities of autonomy, reactivity, pro-activity and sociability. On the other hand, in the strong notion of agent, are defined imminently cognitive characteristics, that can result in the development of a self-consciousness by part of the agent and in the enablement of other valuable properties such as perception, sentimentality and emotions [11. The establishment of this characteristics is an important factor in the contextualization of the designation of intelligent agents in a way to normalize what is in fact and object, and any other form of software based intelligent entities.

The comprehension of these characteristics has to be a analyzed relatively to a Level of Abstraction (LoA) that uniforms them and limit the possibility of relativism on their analysis. Turing first used the notion of LoA to, according to a level established by him, to define intelligence. This concept was used by Floridi and Sander to analyze, according to different LoA the characteristics of intelligent agents before their capacity to undertake moral decisions. Although LoA is a concept derived from Computer Science, more concretely from the disciplines of Formal Methods, that uses discrete mathematics to specify and analyze the behavior of information systems. A LoA consists in a collection of observable, being each one defined by a group of values and results. In other words, before the same entity there exists different LoA that characterize it in a distinct way without adding any type of relativity in the resulting observation. Given a set of values of $\mathrm{X}$ well defined, an observable of the type $\mathrm{X}$ is the variable which response values are contained in X. A LoA consists then in a collection of observable that are considered on the observation. In a less abstract level, in the case of a car, there can be defined different LoA such as of the buyer, mechanic, insurance officer, all of which present different points and characteristics that, even being distinct, do not present relativity 12$]$.

Depending on the LoA, just as an entity can be considered an object or an intelligent agent, defining the proper LoA the properties that defined a MA can be be of use, being for this reason the notion of LoA used by Floridy and Sanders 
to define the criteria which must be included in the LoA of a moral agent. The three criteria considered in this LoA are interactivity (a), autonomy (b) and adaptability (c), being the synchronous existence of these characteristics what enables an intelligent agent to become a MA [12]. In order to better analyze these characteristics, one must specify and adequate them with the definition of an agent and, as well, with the state of the art of the development of intelligent agents, namely:

(a) The base for interaction underlaying this study is related with the capacity of the agent to identify and comunicate with other agents, nevertheless their nature, i.e. wether they might be MA or AM, software based or human beings. It can be related with the reactivity described by Wooldridge, before different stimulus provided by the environment where the agent is based. Comparing with the string definition of agent, one can perceive this as the ability to socialize and relate with another.Taking into consideration this property there are norms developed by the Foundation for Intelligent Physical Agents (FIPA) in order to normalize the communication among agents in different systems and based on different technologies [13] 11.

(b) The autonomy of an agent is a function of its grasp on the universe of discourse and must be in line with its own objectives. A moral agent has the capability to change its state without any external intervention that will force it into a particular line of action. Contrary to an object in Object Oriented Programming, a moral agent is not invoked and in a certain way "forced" to execute a determined action; the agent only performs actions according to its own directives. This characteristic is already considered so essential that exists middleware based on Object Oriented Programming, like the Java Agent DEvelopment Framework (JADE), that protect their agents from remote evocation 13 .

(c) The adaptability of an agent is linked to its capacity to learn and adapt its own behaviors according to the surrounding environment, without external intervention.

Modeling moral agent behavior is in line with the procedures being used to simulate human moral behavior. Although this simulation may provide a better understanding of human ethical choices and give a new perspective on moral in general, the lines under which an agent evolves its moral codes are yet to be set in order to be used as a mean towards building moral agents [14.

\section{Moral Decisions}

Considering the LoA used in the previous section, let us consider an Intensive Care Unity (ICU) with 2 (two) monitoring agents. Both agents interact with the environment reading the patients monitoring data, either it comes from cameras, oxygenation level reading devices or electrocardiograms. In a similar way, both agents can alter their procedures, such as altering oxygenation and temperature levels in the room or warning the medical team of the existence of 
any abnormality. They are also capable to predict future situations, extracting rules from past situations for future use, and to integrate them in the depths of their soul. These agents are ruled by a set of ethic norms, having as their ultimate objective the provision of the best possible service to the patient. Presenting a scenario in this way, are these agents moral agents? According to the LoA of moral agent, one may conclude that yes, they are. Both of them are moral agents, however, one is a human agent, while the other is a software one. In fact, both will be able and probably will commit os ethically dubious, if not incorrect; however, it is clear that contrary to the second case, the responsibility of their actions reflect only upon themselves. In other words, the responsibility in the case of the agent is not so clear to be defined concerning the entity it should reflect, if the agent itself, or its owner, or even other environment input. Its certain that from the developers LoA, an agent is not as independent, proactive, or interactive as it resembles, once he/she set the rules that the agent has to follow. However, taking into consideration its capacity of adaptation, it is expectable that in the short term it may remodel itself into a version completely distinct from the former one. It acts in the same way as a father that educates a son and transmits to him/her his moral code. However, there is always the question: to whom should be inputted the responsibility of their future actions.

Although the enlargement of the moral agent class in order to include the existence of virtual agents which are also moral agents, is not consensual, i.e. it is valid and advisable considering the inevitability of, during one of its learning cycles, a moral decision presents itself to the agent. It is thereby essential to define a set of principles that will allow an improvement of the agent development process, delimitating the frontier of action and principles that ensure, not only in the future, but as well as in the present, that these systems will work in synergies with society.

Although norms and regulations have been made for standardization of agent argumentation and communication, no similar approach has yet to be successful in the definition of properties that are essential for agents to have when taking actions in an areas such as medicine where sometimes little decision may have humungous ethical drawbacks. While a general ethics code was in fact developed by the Association for Computing Machinery (ACM), this code, though comprised of essential points which are essential for any area and technology, is by this reason not specific enough for the needs of agents developer in the healthcare area [15] A set of guidelines and rules must be defined to clearly state the characteristics an intelligent agent must have to be considered moral agent as well as the division of the developers responsibility, and the major role taken by the environment through machine learning techniques.

\section{Modelling Morality with Extended Logic Programming}

With respect to the computational paradigm it were considered extended logic programs with two kinds of negation, classical negation, $\neg$, and default negation, not. Intuitively, not $p$ is true whenever there is no reason to believe $p$ (close world 
assumption), whereas $\neg p$ requires a proof of the negated literal. An extended logic program (program, for short) is a finite collection of rules and integrity constraints, standing for all their ground instances, and is given in the form:

$$
\begin{aligned}
& p \leftarrow p_{1} \wedge \ldots \wedge p_{n} \wedge \text { not } q_{1} \wedge \ldots \wedge \text { not } q_{m} ; \text { and } \\
& ? p_{1} \wedge \ldots \wedge p_{n} \wedge \text { not } q_{1} \wedge \ldots \wedge \text { not } q_{m},(n, m \geq 0)
\end{aligned}
$$

where ? is a domain atom denoting falsity, the $p_{i}, q_{j}$, and $p$ are classical ground literals, i.e. either positive atoms or atoms preceded by the classical negation $\operatorname{sign} \neg$ 16. Every program is associated with a set of abducibles. Abducibles may be seen as hypotheses that provide possible solutions or explanations of given queries, being given here in the form of exceptions to the extensions of the predicates that make the program.

For example, let us suppose that in the KB (Knowledge Base) of the $A g R$ (agent R) the information related to the areas of expertise of the $A g P_{i}$ identified as Peter, is represented by the following program:

$$
\begin{aligned}
& \text { area_of_expertise("Peter", pediatrics). } \\
& \neg \text { area_of_expertise("Peter", oncologist). }
\end{aligned}
$$

If the $\mathrm{KB}$ is questioned if the area of expertise of Peter is pharmacy the answer should be unknown, because there is no information related to that. On other hand, situations of incomplete information may involve different kinds of nulls. The ELP language will be used for the purpose of knowledge representation. One of the null types to be considered stands for an unknown value, a countable one (i.e. it is able to form a one-to-one correspondence with the positive integers). As an example, let us suppose that one of the agents that belong to the agent community $A g P$, at the registration phase, does not specify its interest topics; it just informs that it has interest topics. This means that the interest topics of the agent are unknown:

$$
\begin{aligned}
& \neg \operatorname{skill}(A, B) \leftarrow \text { not } \operatorname{skill}(A, B) \wedge \text { not exception }(\operatorname{skill}(A, B)) . \\
& \text { exception }(\operatorname{skill}(A, B)) \leftarrow \operatorname{skill}(A, \text { something }) . \\
& \text { skill("John", something }) .
\end{aligned}
$$

Another type of null value denotes information of an enumerated set. Following the previous example, suppose that an agent does not give information related to its availability, but its state of affairs is one of the three: uncommitted, committed or in-action:

$$
\begin{aligned}
& \neg \text { availability }(A, B) \leftarrow \text { not availability }(A, B) \\
& \wedge \text { not exception (availability }(A, B)) . \\
& \text { exception(availability(" John", committed)). } \\
& \text { exception(availability(" John", uncommitted)). } \\
& \text { exception(availability("John", in-action)). }
\end{aligned}
$$




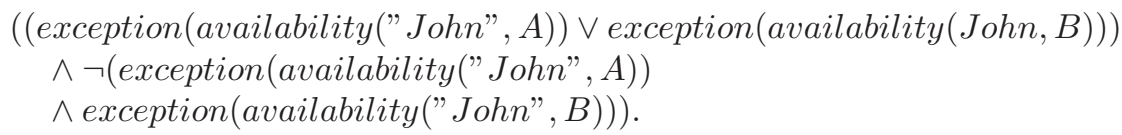

The last statement stands for an invariant that denotes that the agent states of committed, uncommitted and in-action are disjointed.

Therefore, being $\Gamma$ a program in ELP and $g(X)$ a question where $X$ contains variables $X_{1} \wedge \ldots \wedge X_{n}(n \geq 0)$, one gets as an answer:

The answer of $\Gamma$ to $g(X)$ is true iff

$$
g(X) \rightarrow \operatorname{demo}(\Gamma, g(X), \text { true }) .
$$

The answer of $\Gamma$ to $g(X)$ is false iff

$$
\neg g(X) \rightarrow \operatorname{demo}(\Gamma, g(X), \text { false }) .
$$

The answer of $\Gamma$ to $g(X)$ is unknown iff not $\neg g(X) \wedge$ not $g(X) \rightarrow \operatorname{demo}(\Gamma, g(X)$, unknown $)$.

where unknown stands for a truth value in the interval 0...1. Being $\Gamma$ a Program it is possible to define the Minimal Answer Set of $\Gamma(M A S(\Gamma))$ :

$$
\Gamma \vdash s \text { iff } s \in M A S(\Gamma)
$$

where $\Gamma \vdash s$ denotes that $s$ is a logical consequence or conclusion for $\Gamma$.

Being now $A S_{i}$ and $A S_{j}$ two different answer sets of $\Gamma$, being $E_{A S i}$ and $E_{A S j}$, respectively, the extensions of predicates $p$ in $A S_{i}$ and $A S_{j}$, it is defined that $A S_{i}$ is morally preferable to $A S_{j}\left(A S_{i} \prec A S_{j}\right)$ where $\prec$ denotes the morally preferable symbol, if for each predicate $p_{1}$ it exists a predicate $p_{2}$ such that $p_{1}<p_{2}$ and $E_{A S i} \backslash E_{A S j}$ is not empty ( $\backslash$ denotes the difference set operator).

\section{Conclusions}

It is believed that this work is a step in the direction of the final goal of machine ethics, i.e. to create a machine that by itself will follow an ideal ethical principle or set of principles making decisions about possible courses of action it could take. Indeed, it was shown how a machine might abstract an ethical principle from a logical representation of ethical judgments and use that principle to guide its own behavior. A machine that is an explicit ethical agent will be able to calculate the best action in ethical dilemmas using ethical principles. It may represent ethics explicitly and then operate effectively on the basis of this knowledge, here given in terms of logical formulae, understood as productions in a Multi-value Extended Logic Programming Language, as referred to above. On the other hand, and for those that populated the Artificial Intelligence and Logic Programming arenas, it may sanction support for work leading to the development of autonomous intelligent machines that may contribute to improve the lives of human beings. A machine that is an explicit ethical agent will be able to calculate the best action in ethical dilemmas using ethical principles. It will represent ethics explicitly and then operate effectively on the basis of this knowledge. 


\section{References}

1. Deigh, J. (ed.): Ethics and Personality: Essays in Moral Psychology. Chicago University Press (1992)

2. Deigh, J.: The Sources of Moral Agency: Essays in Moral Psychology and Freudian Theory. Cambridge University Press, Cambridge (1996)

3. Andrade, F., Neves, J., Novais, P., Machado, J.: Software agents as legal persons. Virtual Enterprises and Collaborative Networks 149, 123-132 (2004); CamarinhaMatos, LM 18th World Computer Congress, Toulouse, France, August 22-27 (2004)

4. Andrade, F., Neves, J., Novais, P., Machado, J., Abelha, A.: Legal security and credibility in agent based virtual enterprises. Collaborative Networks and Their Breeding Environments 186, 503-512 (2005); CamarinhaMatos, LM 6th Working Conference on Virtual Enterprises SEP 26-28, Valencia, Spain (2005)

5. Machado, J., Alves, V., Abelha, A., Neves, J.: Ambient intelligence via multiagent systems in medical arena. International Journal of Engineering Intelligent Systems, Special issue on Decision Support Systems 15(3), 167-173 (2007)

6. Nwana, H.S.: Software agents: An overview. In: Knowledge Engineering Review, vol. 11, pp. 1-40. Cambridge University Press, Cambridge (1996)

7. Machado, J., Abelha, A., Novais, P., Neves, J., Neves, J.: Improving patient assistance and medical practices through intelligent agents. In: Workshop on Health Informatics, AAMAS 2008 (2008)

8. Miranda, M., Abelha, A., Santos, M., Machado, J., Neves, J.: A group decision support system for staging of cancer. In: Weerasinghe, D. (ed.) Electronic Healthcare, Springer-Verlag, Series Institute for Computer Sciences, Social Informatics and Telecommunications Engineering (2009)

9. Himma, K.E.: Artificial agency, consciousness, and the criteria for moral agency: what properties must an artificial agent have to be a moral agent? In: Ethics and Information Technology. Springer, Heidelberg (2008)

10. Wooldridge, M.J.: Multiagent Systems - A Modern Approach to Distributed Artificial Intelligence. MIT Press, Cambridge (1999)

11. Wooldridge, M., Jennings, N.R.: Intelligent agents: Theory and practice, vol. 10, pp. 115-152. Cambridge University Press, Cambridge (1995)

12. Floridi, L., Sanders, J.W.: On the morality of artificial agents, Hingham, MA, USA, vol. 4, pp. 349-679. Kluwer Academic Publishers, Dordrecht (2004)

13. Bellifemine, F., Caire, G., Greenwood, D.: Developing Multi-Agent Systems with JADE. John Wiley \& Sons, Chichester (2007)

14. Pereira, L.M., Saptawijaya, A.: Modelling morality with prospective logic. In: Neves, J., Santos, M.F., Machado, J.M. (eds.) EPIA 2007. LNCS (LNAI), vol. 4874, pp. 99-111. Springer, Heidelberg (2007)

15. ACM: Code of ethics and professional conduct (January 2009)

16. Analide, C., Abelha, A., Machado, J., Neves, J.: An agent based approach to the selection dilemma in cbr. In: Badica, C., Mangioni, G., Carchiolo, V., Burdescu, D. (eds.) Intelligent Distributed Computing, Systems and Applications. Studies in Computer Science, vol. 162, Springer, Heidelberg (2008) 